\title{
EL AUMENTO DE LA FRAGILIDAD CON EL TAMAÑO EN ESTRUCTURAS DE HORMIGÓN
}

\author{
(THE SIZE EFFECT IN CONCRETE STRUCTURES)
}

Manuel Elices Calafat, Dr. Ingeniero de Caminos

Universidad Politécnica de Madrid. Dpto. de Ciencia de Materiales

ESPAÑA

Fecha de recepción: 26-VI-98

\section{RESUMEN}

En este artículo se comentan dos métodos utilizados para modelizar el proceso de fractura del hormigón; el modelo de la fisura cohesiva y el de la fisura equivalente. A partir de estos modelos se puede predecir cuantitativamente el aumento de la fragilidad de un elemento estructural al aumentar su tamaño, fenómeno conocido como efecto del tamaño.

En la segunda parte se muestran dos aplicaciones de la Mecánica de la Fractura al hormigón; una para el hormigón en masa y otra para vigas débilmente armadas.

\section{SUMMARY}

The cohesive crack model and the equivalent crack are summarized. These models allow quantitative predictions in concrete structures of the increase in brittleness as size increases; the well known size effect.

In the second part, two applications of the cohesive crack model are briefly described; one for plain concrete and the other related to beams with minimum reinforcement.

\section{INTRODUCCIÓN}

Los 50 años de cualquier acontecimiento es siempre una fecha singular y los editores de la revista Informes de la Construcción han decidido conmemorar su singladura de medio siglo con la publicación de un número especial. Agradezco, muy de veras, la invitación de la Directora del Instituto Eduardo Torroja y del Director de la Revista para participar en este evento.

Estas fechas señaladas son un buen motivo para hacer un balance de la actividad desarrollada y la elección del tema, o mejor del material -hormigón o acero-, ha ofrecido pocas dudas porque el acero ha sido objeto de varios resúmenes y el hormigón no. Me asomé a Informes, hace casi 30 años [1], para comentar el fenómeno de la corrosión bajo tensión de las armaduras y hace poco caí en la tentación de inventariar el trabajo realizado sobre este problema complejo, todavía no resuelto satisfactoriamente, con ocasión del homenaje al Dr. Sebastián Feliu [2]. El protagonismo del acero en las estructuras pretensadas quedó reflejado en otro artículo-resumen, publicado hace diez años en Informes, donde se comentaba la patología y la terapéutica de las armaduras activas en el hormigón pretensado [3]. Por el contrario, al hormigón -este material, que siempre nos sorprende- no le había dedicado ningún resumen, aunque contribuí a que Informes le editara dos números, prácticamente monográficos: uno sobre la Fractura del Hormigón [4] y otro sobre Depósitos Criogénicos de hormigón pretensado [5]. En este artículo se resumen varias contribuciones al conocimiento de la fractura del hormigón realizadas durante los últimos diez años por el equipo del 
Departamento de Ciencia de los Materiales de la Escuela de Caminos de la Universidad Politécnica de Madrid.

\section{RELEVANCIA DE LA MECÁNICA DE LA FRACTURA}

Se sabe que las estructuras de hormigón se comportan más frágilmente a medida que su tamaño aumenta. No obstante, se ignora este fenómeno en muchas normas para el proyecto de estructuras y se usan los mismos criterios de diseño para estructuras homotéticas, decisión que no está del lado de la seguridad.

Las investigaciones realizadas en el hormigón, principalmente en la última década, han demostrado que este efecto del tamaño se puede predecir y calcular teniendo en cuenta el proceso de fractura del hormigón. Cuando se sabe modelizar este fenómeno es posible predecir y evaluar la fragilidad de una estructura en función de su tamaño.

Puede pensarse que el aumento de la fragilidad con el tamaño es debido a que un elemento más grande alberga más defectos y, por lo tanto, es más débil. Este efecto -que sin duda puede contribuir- no justifica los resultados experimentales, como se demuestra en el excelente libro sobre la fractura del hormigón escrito por J. Planas y Z.P. Bazant [6]. La verdadera causa de la fragilidad hay que buscarla en el proceso de fractura, ya que este fenómeno -el aumento de la fragilidad con el tamaño- se da también en los materiales idealmente homogéneos; es decir, con defectos de un tamaño que no depende de las dimensiones de la estructura.

En este artículo se resumen dos métodos utilizados para modelizar el proceso de fractura del hormigón desarrollados por el equipo del Departamento de Ciencia de Materiales durante la última década y que proporcionan excelentes predicciones cuando se han comparado con resultados experimentales. En la segunda parte se comentan varias aplicaciones estructurales, tanto en hormigón en masa como en hormigón armado.

\section{MODELIZACIÓN DE LA FRACTURA EN EL HORMIGÓN}

Entre los distintos modelos propuestos para simular la fractura del hormigón, posiblemente el modelo de la fisura cohesiva sea el más general y simple. Parece ser que este modelo es capaz de explicar y de predecir la mayoría de los resultados experimentales obtenidos con probetas de hormigón. Además, este modelo se puede simplificar -dentro de un intervalo de tamaños razonable- aproximándolo mediante una fisura elástica equivalente y su curva $R$ asociada. En estos casos, los modelos basados en dos parámetros, de Bazant y de Jenq-Shah, se pueden considerar un caso particular de esta equivalencia. Finalmente, si el tamaño del elemento considerado es muy grande, todos estos modelos se pueden aproximar utilizando la mecánica de la fractura elástica y lineal [7, 32]. Una exposición detallada de estas equivalencias junto con la estructura jerárquica de estos modelos se puede consultar en [8].

A continuación, los modelos de la fisura cohesiva y su aproximación -la fisura elástica equivalente- se resumen brevemente, destacando cómo se pueden obtener sus parámetros y cómo se pueden aplicar para predecir la fragilidad de las estructuras con el aumento del tamaño.

\subsection{El Modelo de la Fisura Cohesiva}

\section{Conceptos básicos}

Este modelo surgió, a comienzos de los años 60, como una alternativa de la mecánica de la fractura elástica y lineal para eliminar la singularidad tensional que aparece en el fondo de una fisura $[9,10]$. En 1976 Hillerborg lo generalizó, suprimiendo la restricción de que la zona cohesiva fuera pequeña [11] y, desde entonces, el equipo del Departamento de Ciencia de los Materiales de la Escuela de Caminos de Madrid ha ido precisando y generalizando este modelo $[6,12,13,33]$.

El modelo cohesivo, en su versión más simple, supone que:

a) El material, fuera de la fisura, se comporta de forma elástica y lineal. (Se puede generalizar para otros comportamientos).

b) La fisura se inicia en el punto donde la tensión principal máxima alcanza el valor $f_{i}$. La fisura se forma en el plano perpendicular a la dirección de esta tensión.

c) La fisura se propaga cuando las dos caras de este plano se separan. Se supone que entre ellas se transmite una tensión -la tensión cohesiva- que es función de la historia de los desplazamientos relativos entre las dos caras $w$. Para el caso más simple de apertura monótona, se supone que esta tensión $\sigma$ es una función única de $w$;

$$
\sigma=f(w)
$$

Esta función, llamada función de ablandamiento, se supone que es una propiedad del material y tiene el aspecto indicado en la figura 1.

Los parámetros más característicos de esta curva son:

$f_{1}$ : La tensión que se transfiere en el momento de iniciarse la fisura.

$\mathrm{f}_{\mathrm{l}} / \mathrm{w}_{1}$ : La pendiente inicial de la curva (para elementos estructurales fisurados no muy grandes y para elementos no fisurados de cualquier tamaño, estos dos parámetros $-f_{t}$ y $w_{l}$ - son suficientes para predecir la carga máxima $[14$, 15]). 
$\mathrm{w}_{\mathrm{c}}$ : La abertura crítica de la fisura, a partir de la cual no se transfieren tensiones entre las dos caras.

$\mathrm{G}_{\mathrm{F}}$ : La energía específica de fractura. $G_{F}$ es el trabajo, por unidad de área, necesario para separar completamente las dos caras y viene dado por el área encerrada bajo la curva $f(w)$. Por este motivo, $G_{F}$ también es una propiedad del material. (Las medidas experimentales de $G_{F}$ no proporcionan, a veces, un valor constante. Posiblemente estos resultados indiquen que la función de ablandamiento depende, además de $w$, de otras variables. Éste es un tema todavía no resuelto).

La función de ablandamiento para el hormigón se puede aproximar, con resultados satisfactorios, por una función bilineal (como la indicada en la figura 1b). Los cuatro parámetros necesarios para caracterizar esta función se pueden obtener a partir de dos ensayos [16]; el ensayo de tracción indirecta (o brasileño) y el ensayo de la viga entallada. Para este segundo ensayo, que mide $G_{F}$, hay una propuesta de norma RILEM [37], su realización es delicada y en las referencias [17, 18 y 19] se dan indicaciones para la correcta interpretación de los resultados.

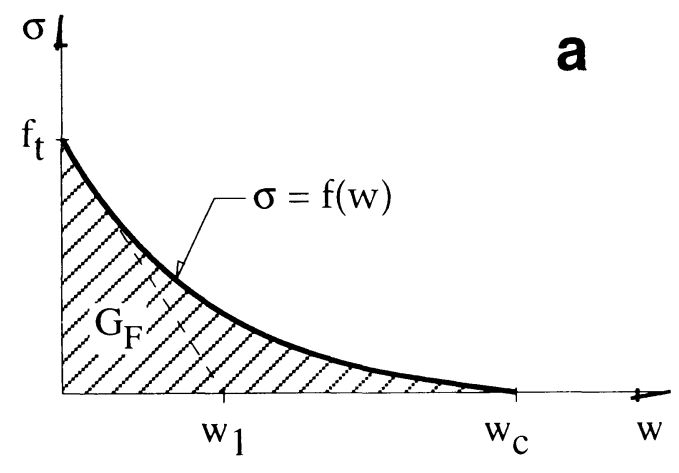

\section{Interpretación del efecto del tamaño}

Dado un elemento estructural sometido a una determinada solicitación es posible calcular la relación entre la carga aplicada y su desplazamiento conjugado ( $u$ otra variable relacionada con él, como la abertura de la fisura, si se trata de un elemento fisurado) aplicando el modelo cohesivo, comentado anteriormente.

El citado equipo del Departamento de Ciencia de Materiales ha desarrollado un programa de cálculo numérico que permite realizar dichas operaciones [20, 33] y obtener, para cada tamaño del elemento estructural, el valor de la carga máxima o de la tensión nominal máxima $\sigma_{N m}$ (calculada dividiendo la carga por una sección transversal convencional).

En la figura 2 se muestra, como ejemplo, la curva del efecto del tamaño para la tensión nominal máxima en una serie de vigas homotéticas de hormigón sometidas a flexión (simplemente apoyadas y con una carga en el centro). En este ejemplo se ha representado la variación del módulo de rotura $\sigma_{R}$ (definido convencionalmente asi; $\sigma_{R}=6 \mathrm{M} / \mathrm{BD}^{2}$,

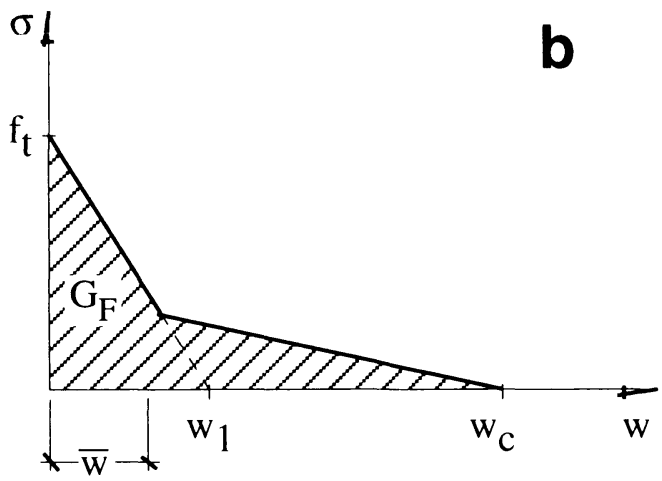

Figura 1.- Función de ablandamiento y sus parámetros: a) Forma general; b) Forma aproximada por dos tramos rectos.

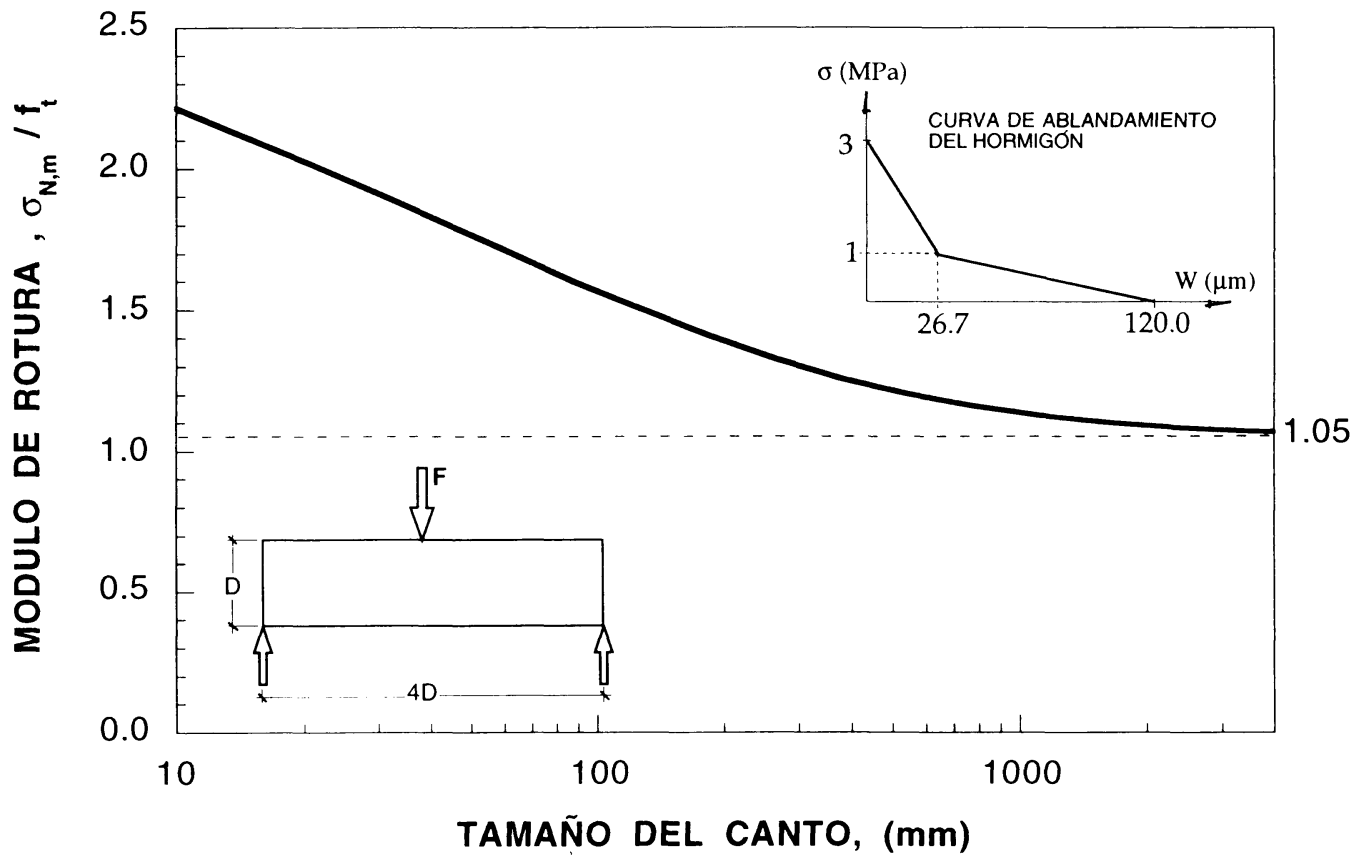
viga de hormigón. 
donde $M$ es el momento en la sección central, $D$ el canto y $B$ el espesor). En este caso, al ser la luz igual a $4 D$, resulta que $\sigma_{N m}=\sigma_{R}$. Las características del hormigón también se indican en la figura. Los resultados muestran claramente que para tamaños grandes $\sigma_{R} / f$ disminuye y tiende al valor teórico aproximado 1.053 [15]. Este efecto reconocido y recogido en algunas normas se explica de una forma sencilla con el modelo de la fisura cohesiva. Para más detalles sobre este tema puede consultarse la citada referencia [15].

En la práctica es deseable disponer de una expresión analítica para poder predecir el aumento de la fragilidad con el tamaño. Para los tamaños y configuraciones usuales de los elementos estructurales de hormigón se puede escribir que la tensión nominal máxima viene dada por [23, $24,25]$ :

$$
\sigma_{N, m}=f_{t} \cdot \psi\left(D / l_{c h}\right)
$$

donde $\psi$ es una función adimensional y la longitud característica $l_{c h}$, se define de la siguiente forma:

$$
1_{c h}=E^{\prime} G_{F} / f_{1}^{2}
$$

J. Planas [26] ha propuesto la siguiente expresión, relativamente simple, que tiene un buen comportamiento asintótico, tanto para tamaños grandes como para tamaños pequeños.

$\sigma_{N, m}=f_{t} \cdot n_{0}\left[\frac{m_{1}+\left(m_{1}+1\right) \sqrt{m_{o} D / l_{c h}}}{m_{1}+\sqrt{m_{0} D / l_{c h}}}+m_{2} \frac{D}{l_{c h}}\right]^{-1 / 2}$ donde $n_{0}, m_{0}, m$, y $m$, son parámetros adimensionales que dependen de la geometría del elemento estructural y del tipo de hormigón considerado. Estos cuatro parámetros se pueden determinar a partir de la información disponible sobre el comportamiento asintótico, como se indica en la citada referencia [26].

En la figura 3 se muestra cómo varía la tensión nominal máxima con el tamaño para una viga fisurada sometida a ilexión. La curva se ha obtenido aplicando la expresión (4). En la misma figura se han representado los resultados experimentales de la tensión nominal máxima en vigas de varios materiales pétreos (caliza, mármol y granito) medidos por varios autores. Como puede observarse, las predicciones de la carga máxima son muy buenas. Muchos ejemplos más avalan la bondad de este modelo para predecir el aumento de la fragilidad con el tamaño en estructuras de hormigón.

\subsection{El Modelo de la Fisura Equivalente}

Los cálculos basados en el modelo de la fisura cohesiva no son sencillos y requieren el uso de técnicas especiales de cálculo numérico [33]. Por otra parte, se dispone de un arsenal de técnicas simples para analizar una fisura en régimen elástico y lineal [7]. Estos dos motivos hacen deseable encontrar un modelo intermedio que permita analizar una fisura cohesiva utilizando las técnicas que se aplican a una fisura elástica lineal.

El modelo de la fisura equivalente nació con este propósito. Esta aproximación del modelo de la fisura cohesiva no

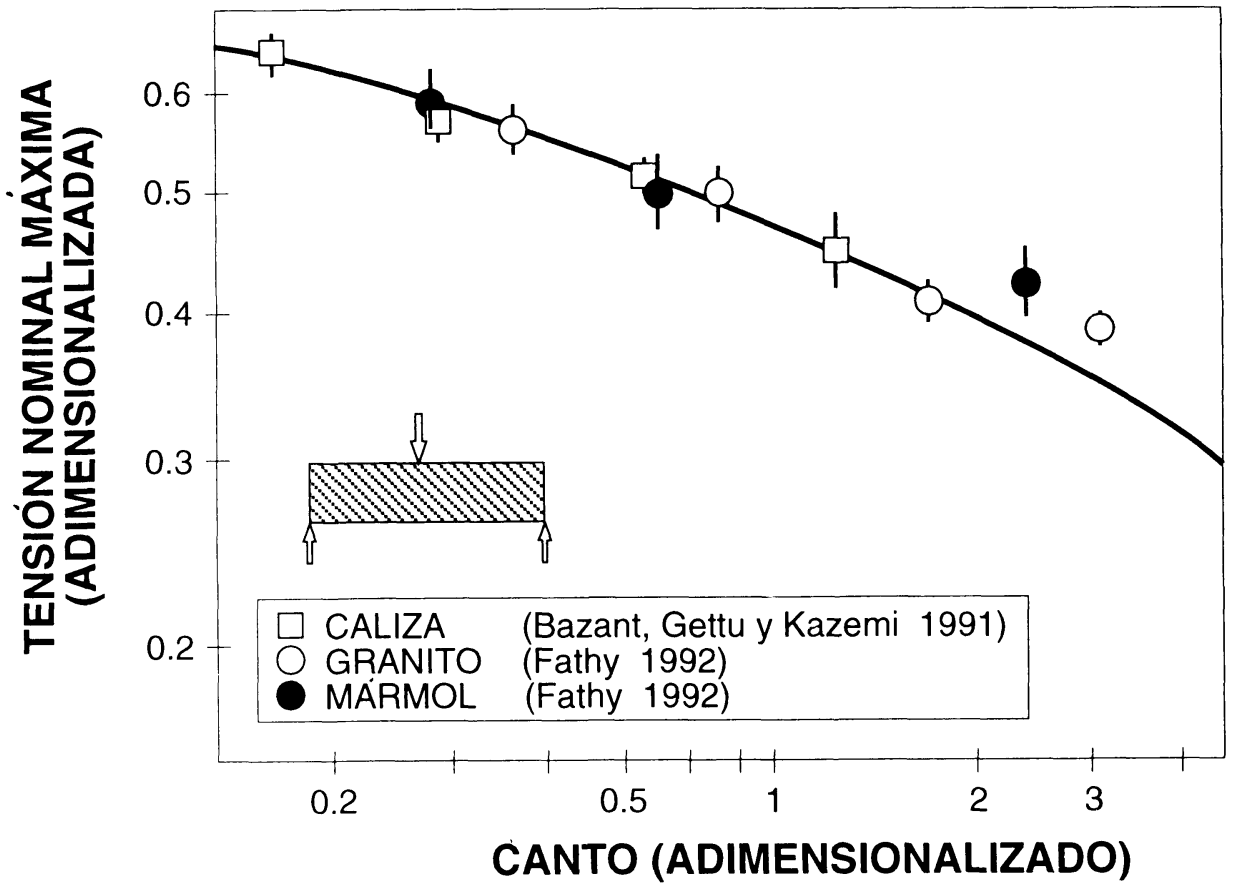

Figura 3.- Disminución de la tensión nominal máxima con el tamaño. Predicciones teóricas, según la fórmula (4), y resultados experimentales en materiales pétreos [26] 
siempre es legítima y, en cada caso, debe justificarse. El concepto de la fisura equivalente y los detalles del modelo pueden consultarse en $[27,28]$. Otro aspecto interesante de este modelo es que permite considerar los modelos de Bazant [29] y de Jenq-Shah [30] (de dos parámetros) como casos particulares de esta equivalencia, proporcionando así una visión jerarquizada de los distintos modelos de fractura del hormigón dentro del marco de la fisura cohesiva [8].

El concepto de la fisura equivalente se puede comentar a través del siguiente ejemplo. Para este propósito se ha seleccionado una equivalencia particular; la $P-C M O D$, carga-abertura de la grieta (crack mouth opening displacement). En la figura 4, dos probetas fisuradas con la misma geometría están cargadas con control del $C M O D$. La probeta $a$ está fabricada con un material cohesivo y la probeta $b$ (la equivalente) con un material elástico lineal. Para un mismo valor del $C M O D$ las cargas que se medirían en las dos probetas, $P$ y $P_{e q}$, serían en general distintas, pero podemos forzar que ambas cargas sean iguales $\left(P=P_{e q}\right)$ eligiendo en la probeta elástica una determinada longitud de fisura $a_{e q}$ y una resistencia específica $R_{e q}$ adecuada. (Esto se indica en la parte inferior de la figura).

Con este procedimiento obligamos a que ambas probetas exhiban el mismo comportamiento $P$-CMOD pero, en general, la equivalencia termina aquí. Los campos de tensiones, por ejemplo, a lo largo del ligamento no se parecen en nada, como se muestra en la figura 4. El precio que se paga por la equivalencia es que el material elástico lineal no tiene una resistencia a la fractura constante $\left(G_{F}\right.$ es constante tanto en un material cohesivo como en uno elástico y lineal). Ahora la resistencia depende del tamaño de la grieta y se genera una curva $R-\Delta a$. Esta curva, en general, no es una propiedad del material y depende de la geometría y del tamaño de la probeta. En rigor, la fisura equivalente tiene poca capacidad predictiva porque sólo
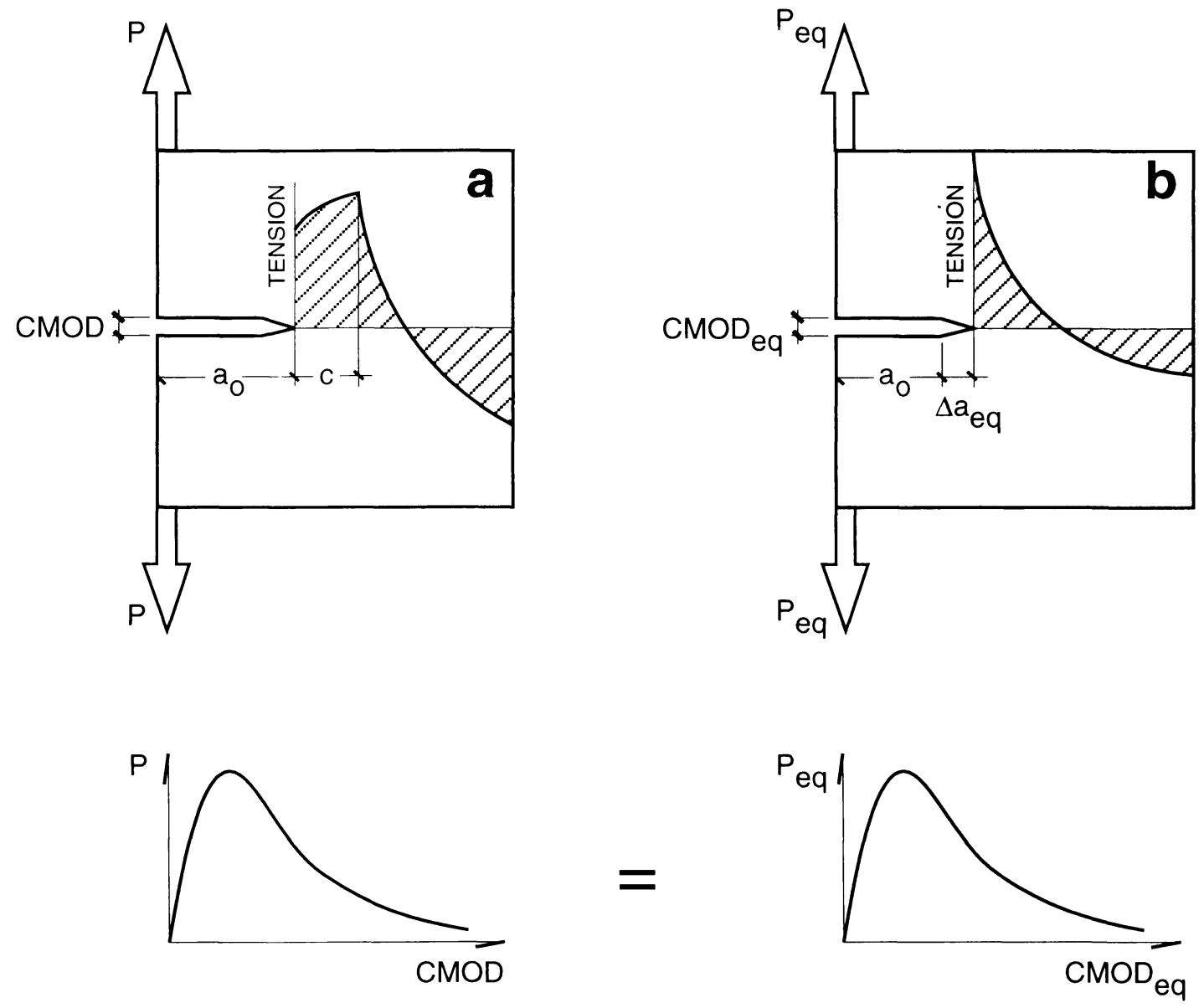

Figura 4.- Ejemplo del concepto de fisura equivalente. 
predice lo que ya sabemos. No obstante, en muchos casos las curvas $R-\Delta a$ para ciertos tamaños y geometrías son prácticamente las mismas y, en estas circunstancias, se puede utilizar la equivalencia con provecho como se muestra en el siguiente ejemplo.

En la figura 5 se han calculado las curvas $P-C M O D$ (la representación en la figura es adimensional) para dos paneles fisurados sometidos a tracción uniforme. La figura 5a es para un panel con una anchura $D=l_{c h}$ y una fisura profunda $(a / D=0,5)$ y la figura $5 b$ es para un panel mucho mayor, $D=3 l_{c h}$, y una fisura más superficial $(a / D=0,1)$. Para este cálculo se ha utilizado la curva $R-\Delta a$ obtenida para una viga fisurada, simplemente apoyada $y$ con una carga en el centro utilizando una equivalencia $P$-CMOD. (El canto de la viga era $D=l_{c h}$ y la profundidad de la fisura $a=0,5 D$ ). En las figuras también se han representado los resultados "experimentales" (obtenidos mediante cálculo numérico a partir del modelo cohesivo). Se aprecia claramente que las predicciones utilizando la fisura equivalente para el panel pequeño son excelentes (aunque se ha usado una curva $R-\Delta a$ teóricamente inco-
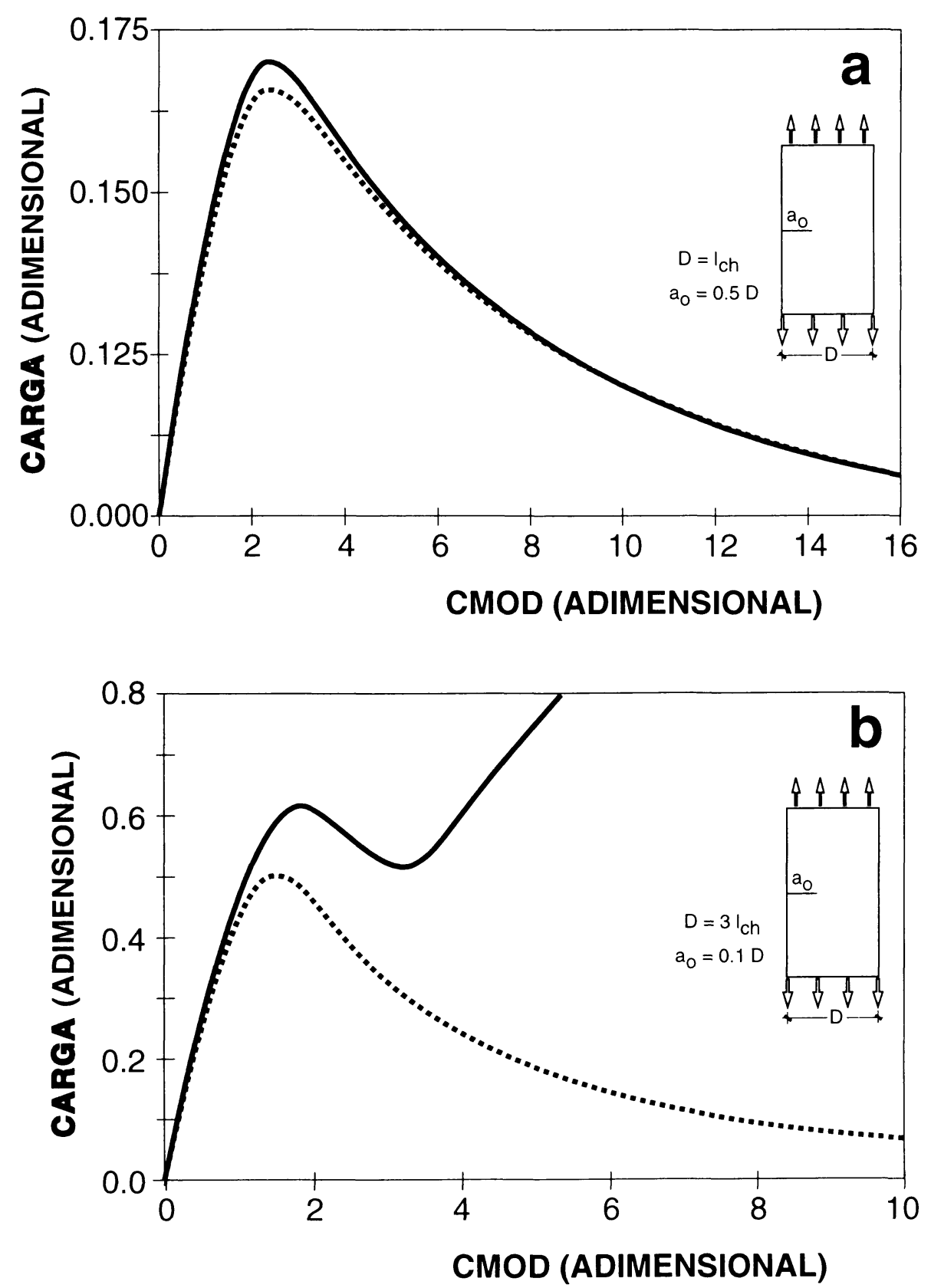

Figura 5.- Curvas P-CMOD (adimensionales) para paneles fisurados obtenidas a partir de una curva $R$ - $\Delta a$ correspondiente a una viga fisurada.

Las curvas continuas son predicciones teóricas basadas en el concepto de fisura equivalente y' las curvas de trazos son resultados "experimentales". 
rrecta) mientras que las predicciones para el panel grande son inadmisibles.

Como se ha mencionado al principio de esta sección, los modelos de Bazant y de Jenq-Shah pueden considerarse aproximaciones del tipo de la fisura elástica equivalente. El modelo de Bazant es una equivalencia $P_{\max }=P_{\text {max }, e q}$, para cada tamaño $D$ y el de Jenq-Shah también es otra equivalencia basada en la carga máxima. Los detalles pueden verse en las referencias $[8,27,28,31]$.

En general, para los tamaños usuales de las probetas de hormigón, las predicciones del efecto del tamaño basadas en el modelo de la fisura equivalente son bastante aceptables y los resultados obtenidos aplicando equivalencias P-CMOD (o similares), o bien a través de los modelos de Bazant o de Jenq-Shah, son parecidos, como se muestra en otro trabajo de J. Planas y el autor [24].

Es de notar que la equivalencia es exacta en el límite de grandes tamaños, en los que el tamaño de la zona cohesiva, $C$, es mucho menor que cualquier dimensión representativa de la estructura $D$. De hecho, uno de los resultados del estudio asintótico realizado por J. Planas y el autor [21, 22] demuestra que "para un elemento fisurado (con una fisura inicial $a_{0}$ ) cualquier campo lejano (de tensiones o desplazamientos) se puede aproximar por el correspondiente campo de una fisura elástica y lineal de tamaño $a_{0}+\Delta a_{c o} . "$, donde $\Delta a_{\infty}$ es la extensión equivalente de fisura para $D / C \rightarrow \infty$. El cálculo de $\Delta a_{\propto}$ se indica en los citados artículos donde, además, se demuestra que $\Delta a_{\infty_{c}}$, el valor de $\Delta a_{\infty}$ en el momento de alcanzarse la carga máxima, es mayor o igual que $\pi w_{c}^{2} E^{\prime} / 32 G_{F}$, siendo $E^{\prime}$ el módulo de elasticidad generalizado del material. En posteriores trabajos se obtuvo que el valor real de $\Delta a_{\infty_{c}}$ es muy próximo a la cota superior para las curvas de ablandamiento más usuales.

\section{ALGUNAS APLICACIONES}

Para finalizar este breve resumen se han incluido dos aplicaciones: La primera, es para resaltar que la energía específica de fractura $G_{F}$ es otra propiedad de los hormigones a tener en cuenta y que no es función solamente de otras propiedades ya conocidas, como el módulo de elasticidad $E$, o la resistencia a compresión $f_{c}$. En el ejemplo se pone de manifiesto que dos hormigones con las mismas propiedades convencionales -es decir igual $E$ y $f_{c}$-pueden tener frente a ciertas acciones un comportamiento totalmente distinto. El segundo ejemplo es una aplicación al hormigón armado de las ideas expuestas en el apartado anterior; en él se justifican -basándose en la mecánica de la fracturaalgunas expresiones empíricas utilizadas en el diseño de vigas con armadura mínima.

\subsection{Relevancia de la Resistencia Específica de Fractu- ra, $G_{F}$}

La importancia de este nuevo parámetro, $G_{F}$, se puede apreciar cuando se analizan situaciones donde el hormigón rompe frágilmente, como en el siguiente ejemplo [34]: Un fabricante de pilotes de hormigón armado detectó roturas frágiles en algunos pilotes de una de sus obras (obra A). El hormigón utilizado tenía las mismas características que el de otra obra (obra B) que daba buenos resultados y, además, el diseño de los pilotes también era el mismo. Las propiedades mecánicas de los dos hormigones — medidas a través de sus módulos de elasticidad y de sus resistencias a la compresión y a la tracción- eran prácticamente las mismas o, quizás, mejores para el hormigón $\mathrm{A}$, como se muestra en la tabla 1 ,

El análisis tradicional difícilmente podía explicar cuantitativamente la diferencia de comportamiento entre los dos hormigones porque las resistencias del hormigón frágil $A$ nunca quedaron por debajo de los valores del hormigón $B$, que se comportaba bien.

Los valores de la resistencia a la fractura $G_{F}$ proporcionaron la clave para analizar estas diferencias. Los valores que se midieron, indicados también en la tabla 1 , muestran claramente que el hormigón $A\left(G_{F}=103 \mathrm{~J} / \mathrm{m}^{2}\right)$ era mucho más frágil que el hormigón $B\left(G_{F}=151 \mathrm{~J} / \mathrm{m}^{2}\right)$. Estos datos permitieron actuar sobre los parámetros que influyen en los valores de $G_{F}$-en este caso, el tipo de árido y la adherencia entre el árido y la matriz- y aumentar su valor hasta conseguir un hormigón con un comportamiento aceptable para la fabricación de pilotes.

La introducción de este nuevo parámetro, $G_{F}$, permitió identificar otra propiedad del hormigón, la tenacidad, y cuantificarla.

\section{TABLA 1}

Propiedades de los hormigones A y B

\begin{tabular}{|c|c|c|c|c||}
\hline Hormigón & $\begin{array}{c}\text { Mod. Elástico } \\
\text { (GPa) }\end{array}$ & $\begin{array}{c}\text { R. Compresión } \\
\text { (MPa) }\end{array}$ & $\begin{array}{c}\text { R. Tracción } \\
\text { (MPa) }\end{array}$ & $G_{1}$ \\
\hline A & 48 & 57 & 5,7 & 103 \\
\hline B & 36 & 44 & 4,5 & 151 \\
\hline
\end{tabular}




\subsection{Vigas de Hormigón con Armadura Mínima}

El segundo ejemplo está relacionado con la cuantía de la armadura mínima. Algunas normas recomiendan una cuantía que no depende del tamaño del elemento estructural y, por consiguiente, no tienen en cuenta el aumento de la fragilidad con el tamaño. Otras normas sugieren una cuantía -basada en argumentos empíricos- que aumenta con el tamaño. El modelo de fractura del hormigón, basado en el concepto de fisura cohesiva, permite calcular una cuantía mínima que depende del tamaño y, además, de la adherencia entre el acero y el hormigón, de la tensión de cedencia del acero y del espesor del recubrimiento de las armaduras.
El concepto de armadura mínima se indica en la figura $6 a$ : Al ensayar a flexión en tres puntos una viga débilmente armada y registrar la carga en función del desplazamiento asociado, se pueden observar los tres tipos de comportamiento indicados en la citada figura, donde la resistencia para gandes desplazamientos es debida exclusivamente a la contribución de la armadura. Si esta resistencia es inferior al pico de la carga máxima se dice que el comportamiento de la viga es frágil y, al revés, dúctil cuando es mayor. La cuantía que proporciona la misma resistencia es la suantía mínima.

Cuando la viga se rompe por una sola grieta central es posible analizar el proceso utilizando el modelo de la fisura
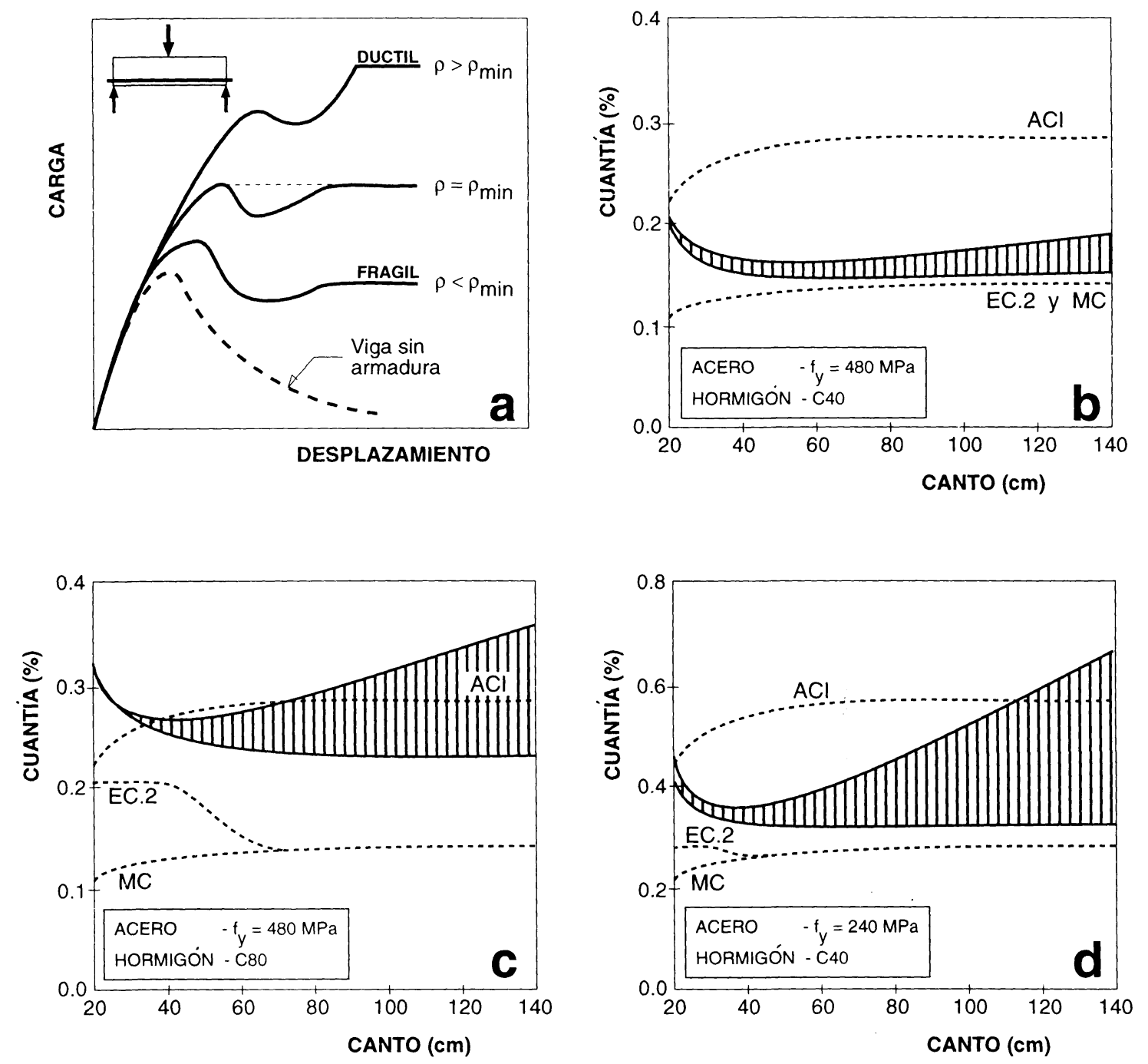

gura 6.- El efecto del tamaño en la cuantía de la armadura minima. a) concepto de armadura minima; b) cuantía en función del canto para un rmigón tipo $(40 ; c)$ cuantia en función del canto para un hormigón tipo C80; d) cuantia en función del canto para un acero con $f=240 \mathrm{MPa}$. Lineas ntinuas, predicciones teóricas. Lineas de puntos, recomendaciones según normas: MC (Model Code) EC.2 (Eurocode 2) y ACI (ACI-3/8 Code). 
cohesiva y calcular la cuantía mínima. Los detalles de estos cálculos pueden verse en $[35,36]$ y solamente algunos resultados, relacionados con los valores de la cuantía mínima, se comentan a continuación.

En la figura $6 \mathrm{~b}$ se muestran los valores de la cuantía mínima en función del canto de la viga según varias normas (CEB-FIP Model Code, ACI-318 Code y Eurocode 2) junto con los valores calculados a partir del modelo de la fisura cohesiva. Los valores de las normas se han obtenido por procedimientos clásicos (análisis límite) y no exhiben ningún efecto de tamaño. (La variación con el canto es aparente porque en todos los casos se considera el mismo recubrimiento, en vez de variarlo proporcionalmente al canto). Las curvas teóricas se han calculado para dos casos extremos de adherencia entre la armadura y el hormigón, es de esperar que en la práctica las curvas reales se situen entre los dos. Se ha seleccionado un hormigón del tipo C40, un acero de límite elástico $f_{y}=480 \mathrm{MPa}$ y un recubrimiento de $4 \mathrm{~cm}$ en todos los ejemplos. Los valores del Eurocódigo y del Model Code son parecidos a las predicciones teóricas, mientras que el código ACI proporciona valores más conservadores.

La figura 6c muestra el efecto producido al considerar un hormigón de mayor resistencia (tipo $\mathrm{C} 80$ ). Los valores recomendados por los códigos son, prácticamente, los mismos, con la excepción del Eurocódigo para cantos inferiores a $50 \mathrm{~cm}$. Por el contrario, las predicciones teóricas indican valores más altos de la cuantía mínima; las recomendaciones del $\mathrm{ACI}$ ya no son tan conservadoras y las otras normas están del lado de la inseguridad.

Por último, en la figura $6 \mathrm{~d}$ se muestra la influencia del límite elástico de la armadura al considerar un acero con la mitad del límite elástico, $f_{y}=240 \mathrm{MPa}$. Los resultados indican que las cuantías, $\rho$, prácticamente se han multiplicado por dos, para mantener constante el producto $\rho f_{1}$. (Nótese que la escala vertical de $6 \mathrm{~d}$ es distinta). Para cantos grandes la influencia de la adherencia entre el hormigón y la armadura es importante, mostrando de nuevo que se pueden dar circunstancias en las que los códigos recomiendan valores que no están del lado de la seguridad. Gran parte de estos resultados teóricos se han comprobado experimentalmente en un extenso plan de ensayos cuyos detailes aparecen en las citadas referencias $[35,36]$.

Aunque en algunos casos el ahorro que se puede conseguir no justifique la utilización de procedimientos más elaborados, es interesante resaltar que con las fórmulas muy simples no siempre se está del lado de la seguridad y, lo que es más importante, se dispone de una herramienta -la Mecánica de la Fractura- que permite dar una interpretación física a los términos empíricos con la que se puede valorar su importancia y conocer su significado.

\section{AGRADECIMIENTOS}

El autor desea expresar su agradecimiento a todos los componentes del equipo del Departamento de Ciencia de Materiales de la Escuela de Caminos, sin cuya cooperación no se hubiera podido llevar a cabo este trabajo y muy especialmente a los profesores Jaime Planas y Gustavo Guinea por sus seminales aportaciones.

Esta investigación se ha podido realizar gracias a la financiación de la CICYT a través de los proyectos MAT971007-C02.02 y 95.0091.OP02.

\section{BIBLIOGRAFÍA}

[1] POZO F. del, ELICES M., CLIMENT J. y BARBA C. (1970). Corrosión bajo tensión en aceros de pretensado Informes de la Construcción 216, 57-69.

[2] ELICES M. (1990). Aspectos de la corrosión en ingeniería civil: Veinte años de experiencias en corrosión bajo tensión. En Retrospectiva de los Estudios de Corrosión en España en el Periodo 1960-1990. CENIM (CSIC), 279-298.

[3] ELICES M. (1988). Patología y terapéutica de las armaduras activas en el hormigón pretensado. Informes de la Construcción 396, 5-18.

[4] ELICES M. (1985). Fractura del hormigón: Métodos de cálculo numérico. Informes de la Construcción 37 (372), 5-18.

[5] EliCES M. y PLANAS J. (1989). Análisis de las especificaciones de los materiales utilizados para el hormigón pretensado a bajas temperaturas. Informes de la Construcción 40(400), 35-42.
[6] BAZANT Z.P. y PLANAS J. (1998). Fracture and size effect in concrete and other quasibrittle materials. CRC Press. London. New York.

[7] ELICES M. (1997). Mecánica de la Fractura. Escuela Técnica Superior de Ingenieros de Caminos. Universidad Politécnica. Madrid.

[8] ELICES M. y PLANAS J. (1996). Fracture mechanics parameters of concrete: An overview. Advanced Cement Based Materials 4, 116-127.

[9] DUGDALED.S. (1960). Yielding of steel sheets containing slits. Journal of the Mechanics and Physics of Solids $\mathbf{8}$, 100-108.

[10] BARENBLATT G.J. (1962). The mathematical theory of equilibrium crack in brittle fracture. Advanced Applied Mechanics 7, 55-125.

[11] HILLERBORG A, MODÉER M.,PETERSSONP.E. (1976). 
Analysis of crack formation and crack growth in concrete by means of fracture mechanics and finite elements. Cement and Concrete Research 6, 773-782.

[12] ELICES M., PLANAS J. (1989). Material models. En Fracture Mechanics of Concrete Structures; Elfgren L., Ed.; Chapman \& Hall: London, 16-66.

[13] ELICES M., PLANAS J., GUINEA G.V. (1993). Modeling cracking in rocks and cementitious materials. En Fracture and Damage of Concrete and Rock; Rossmanith H.P., Ed.; E \& FN Spon: London, 3-33.

[14] GUINEA G.V., PLANAS J., ELICES M. (1994). Correlation between the softening and the size effect curves. En Size Effect in Concrete Structures; Mihashi H.; Okamura H.; Bazant Z.P., Eds.; E. \& FN Spon: London, 233-244.

[15] PLANAS J., GUINEA G.V. Y ELICES M. (1995). Rupture modulus and fracture properties of concrete. En Fracture Mechanics of Concrete Structures I; Wittmann F.H., Ed.; Aedification Publishers: Freiburg, Germany, 95-110.

[10] GUINEA G.V., PLANAS J. Y EliCES M. (1994). A general bilinear fit for the softening curve of concrete. Materials and Structructures 27, 99-105.

[17] GUINEA G.V., PlanAS J. Y EliCES M. (1992). Measurement of the fracture energy using three-point bend tests: 1. Influence of experimental procedures. Materials and Structructures 25, 212-218.

[18] Planas J., EliCES M. Y GUINEA G.V. (1992). Measurement of the fracture energy using three-point bend tests: 2. Influence of bulk energy dissipation. Materials and Structructures 25, 305-312.

[19] ELICES M., GUINEA G.V. Y PLANAS J. (1992) Measurement of the fracture energy using three-point bend tests: 3. Influence of cutting the P-d tail. Materials and Structures 25, 327-334.

[20] PLANAS J. (1988).Splitting-lab. Informe interno del Departamento de Ciencia de Materiales. E.T.S. Ingeniero de Caminos. Universidad Politécnica de Madrid.

[21] PLANAS J. Y ELICES M. (1992). Asymptotic analysis of a cohesive crack: 1 . Theoretical background. International Journal of Fracture 55, 153-177.

[22] PLANAS J. Y ELICES M. (1993). Asymptotic analysis of a cohesive crack: 2 . Influence of the softening curve. International Journal of Fracture 64, 221-237.

[23] PLANAS J. Y ELICES M. (1989). Size effect in concrete structures: Mathematical approximations and experimental validation. En Cracking and Damage, Strain Localization and Size Effect, Mazars J. y Bazant Z.P., Eds.; Elsevier: London, 462-476.

[24] PLANAS J. Y ELICES M. (1990). Fracture criteria for concrete: Mathematical approximations and experimental validation. Engineering Fracture Mechanics 35, 87-94.
[25] PLANAS J. Y ELICES M. (1991). Nonlinear fracture of cohesive materials. International Journal of Fracture 51, 139-157.

[26] PLANAS J., GUINEA G.V. Y ELICES M. (1997). Generalized size effect equation for quasibrittle materials. Fatigue Fract. Engng. Mater. Struct. 20(5), 671-687.

[27] ELICES M. Y PLANAS J. (1993). The equivalent elastic crack: 1. Load-Y equivalences. International Journal of Fracture 61, 159-172.

[28] PLANAS J., ELICES M. Y RUIZ G. (1993). The equivalent elastic crack: 2. X-Y equivalences and asymptotic analysis. International Journal of Fracture 61, 231-246.

[29] BAZANT Z.P., KIM J.K. Y PFĖIFFER P.A. (1986) Nonlinear fracture properties from size effect tests. $J$. Struct. Eng. ASCE 112, 289-307.

[30] JENQ Y.S. Y SHAH S.P. (1985). Two-parameter fracture model for concrete. J. Engng. Mech. Div. 111, 12271241 .

[31] ELICES M. Y PLANAS J. (1992). Size effect in concrete structures: An R-curve approach. En Applications of Fracture Mechanics to Reinforced Concrete, Carpinteri A. Ed.; Elsevier Applied Science: London, 169-200.

[32] ELICES M., LLORCA J. E INGRAFFEA A.R. (1985). Fractura del hormigón en régimen elástico y lineal. Informes de la Construcción 37(372), 19-33.

[33] PLANAS J., GUINEA G.V. Y EliCES M. (1998). Integral equation method for modeling cracking in concrete. En Computational Fracture in Concrete Technology, Aliabadi M.H. y Carpinteri A. Eds.; Computational Mechanics Publications: Southampton (en prensa).

[34] ELICES M., GUINEA G.V. Y PLANAS J. (1992) Choosing the right concrete for piles: An application in concrete fracture mechanics. En Fracture Mechanics of Concrete Structures, Bazant Z. Ed.; Elsevier Applied Science: London and New York, 782-787.

[35] RUIZ G. (1996). El efecto de escala en vigas de hormigón débilmente armadas y su repercusión en los criterios de proyecto. Tesis Doctoral. E.T.S. Ingenieros de Caminos. Universidad Politécnica. Madrid.

[36] RUIZ G., ELICES M. Y PLANAS J. (1998). Size effect and bond-slip dependence of lightly reinforced concrete beams. En Fracture of Concrete, Carpinteri A. Ed. (ESIS TC9) (en prensa).

[37] RILEM: (1995). (Draft Recommendation TC-50 FMC) Determination of the fracture energy of mortar and concrete by means of three-point bend tests on notched beams. Materials and Structures 18, 285-290. 